\title{
Droplet arrangement and coalescence in diverging/converging microchannels
}

\author{
Bibin M. Jose $\cdot$ Thomas Cubaud
}

Received: 15 September 2011 / Accepted: 7 November 2011/Published online: 22 November 2011

(C) Springer-Verlag 2011

\begin{abstract}
We experimentally examine the dynamics of droplet assembly and recombination processes in a twodimensional pore-model system. Monodisperse trains of droplets are formed by focusing streams of immiscible fluids into a square microchannel that is connected to a diverging/ converging slit microfluidic chamber. We focus on the limit of dilute emulsions and investigate the formation and stability of crystal-like structures when droplets are hydrodynamically coupled in the chamber. The minimal distance between droplets and the spread of droplet lattices are measured as a function of initial control parameters and the relationship between droplet velocity and trajectory is discussed. We demonstrate that the onset of coalescence depends on both the capillary number based on the viscosity of the external phase and the droplet concentration. The draining time of the thin film between droplets in apparent contact is found to depend on fluid characteristics. Such property allows us to examine the crossover between noncoalescing and coalescing droplet microflows by varying the residence time of the dispersion in the microfluidic chamber. This work characterizes droplet interaction and coalescence phenomena during multiphase transport in a simple extensional microgeometry.
\end{abstract}

Keywords Multiphase flow Pore $\cdot$ Emulsion . Flow pattern - Lattice $\cdot$ Hydrodynamic coupling

B. M. Jose · T. Cubaud ( $\square)$

Mechanical Engineering Department,

Stony Brook University, Stony Brook, NY 11794, USA

e-mail: thomas.cubaud@stonybrook.edu

\section{Introduction}

Liquid/liquid dispersions have a broad range of applications in material synthesis and petroleum engineering (Larson 1999; Leal-Calderon et al. 2007). In the context of immiscible two-fluid flows in porous media, recombination processes between droplets can significantly alter flow behavior. Although droplet coalescence in air is relatively well understood (Eggers 1997; Wu et al. 2004; Rabe et al. 2010), less is known about droplet merging in flowing emulsions (Yoon et al. 2005). For instance, two droplets in air coalesce upon contact but this process is delayed in viscous environments due to the presence of a thin liquid film between droplets (Jones and Wilson 1978; Aarts and Lekkerkerker 2008). As a result, two droplets, which appear to make contact with one another in an external liquid phase, do not necessarily coalesce even in the absence of surfactant. In confined systems, variations and irregularities in the flow geometry introduce additional complexities for predicting and modeling droplet interactions and coalescence phenomena during multiphase transport at the small-scale.

Microfluidic devices are useful for generating and manipulating arrays of monodisperse droplets. The flow combination of two immiscible fluids in microgeometries produces many flow patterns (Thorsen et al. 2001; Dreyfus et al. 2003; Hashimoto et al. 2008; Teh et al. 2008; Cottin et al. 2011). Microfluidic droplets facilitate a variety of controlled operations and can be used as microreactors for encapsulating cells and chemicals (Brouzes et al. 2009; Gu et al. 2011; Schultz and Furst 2011). Numerous methods based on channel designs have been developed to produce, transport, break, merge, and store droplets in microsystems (Anna et al. 2003; Link et al. 2004; Tan et al. 2007; Bremond et al. 2008; Cubaud 2009; Boukellal et al. 2009). An important microfluidic component consists of a chamber 
made of a planar microchannel connected to multiple ports. A recent theoretical study has shown the possibility to assemble complex structures in a chamber using elaborated time-dependent flows (Schneider et al. 2011). The formation of compact three-dimensional droplet arrays was achieved by controlling the ratio of the chamber height to the droplet diameters in conjunction with several small inlet channels (Hatch et al. 2011). At the transition between a single inlet channel and a wide two-dimensional cell, droplets having a very low interfacial tension with the continuous phase can stretch normal to the main flow direction and breakup into complex arrays (Hashimoto et al. 2008). Overall, chambers are useful for manipulating microfluidic droplets and provide insights for displacing immiscible fluids in porous media.

In this work, we study the flow behavior of droplets as they traverse a microfluidic chamber designed as a slit diverging/converging channel. The flow geometry represents a simplified model system of a two-dimensional pore with practically "stagnant pockets". This type of interconnected pore is often encountered in natural porous matrices (Bear 1972). The extensional geometry also offers new functionalities for probing multiphase flows. For instance, separated flows (i.e., core-annular flows) can experience a significant lubrication failure in a planar flow cell (Darvishi and Cubaud 2011) and dispersed flows of droplets can form an effective jet or "spray" of droplets (Cubaud and Mason 2007). In this study, we examine drop hydrodynamic coupling and the relationship between dispersed and separated flows with high-viscosity fluids in microfluidic chambers.

The article is organized as follows. First, we characterize the microfluidic design, apparatus, and physical properties of fluids used in the study. Continuously focusing immiscible fluid streams into a square channel generates regular trains of monodisperse droplets upstream from the chamber. As this method sets the initial flow conditions prior to the pore, we determine the relationships between droplet size, distance, and velocity in the square channel as a function of flow rates of injection and fluid properties. Downstream, droplets enter the chamber and form a variety of compact lattices. The central part of this paper is devoted to examining the morphology and dynamics of these arrangements. The morphological study includes discussions of the evolution of the droplet distance and the spread of the effective droplet spray in the chamber. Then, we investigate the dynamics of droplet assembly by recording and comparing droplet velocities and residence times in the cell with theoretical predictions. The final part deals with the recombination processes occurring in various droplet lattices. We measure the critical droplet concentration for the onset of coalescence as a function of the capillary number and we demonstrate that the time required for the intercalating film between two nearby droplets to drain and permit coalescence is proportional to the viscous-capillary time-scale based on the viscosity of the external fluid.

\section{Experimental setup}

The microfluidic platform consists of a double-side-polished silicon piece sandwiched between two borosilicate glass slides. Microchannels are etched through the entire height of the silicon wafer $(h=250 \mu \mathrm{m})$ using deep reactive ion etching. Glass pieces are sealed on each side of silicon using anodic bonding. This method is used to make high-pressure, non-deformable microflow devices having the top and bottom walls made of glass and the sidewalls made of silicon. The fluidic system is composed of four consecutive elements including: (1) a right angle focusing section with square microchannels to generate arrays of monodisperse droplets, (2) a long inlet square microchannel of width $h$ and length 20h, (3) a diamond-shaped diverging-converging slit microfluidic chamber of width $w=20 h$, and (4) an outlet square microchannel (Fig. 1). The large chamber aspect ratio, $w / h=20$, is chosen to provide a two-dimensional flow approximation (i.e., HeleShaw flow). The microfluidic module is placed on top of an inverted microscope, which is equipped with a high-speed camera (Redlake, HG-100 K), and a fiber-optic bundle connected to a $150 \mathrm{~W}$ halogen bulb is placed above the microchannel to provide a collimated light source. Droplets are generated by introducing the liquid 1 ( $L 1)$ of viscosity $\eta_{1}$ into the central channel with a volumetric flow rate $Q_{1}$ and the liquid 2 (L2), having a viscosity $\eta_{2}$, into the sidechannels with a total flow rate $Q_{2}$. Each channel is fed independently with fluids that are injected into the device using high-pressure syringe pumps. The role of the long inlet square microchannel is to decorrelate droplet

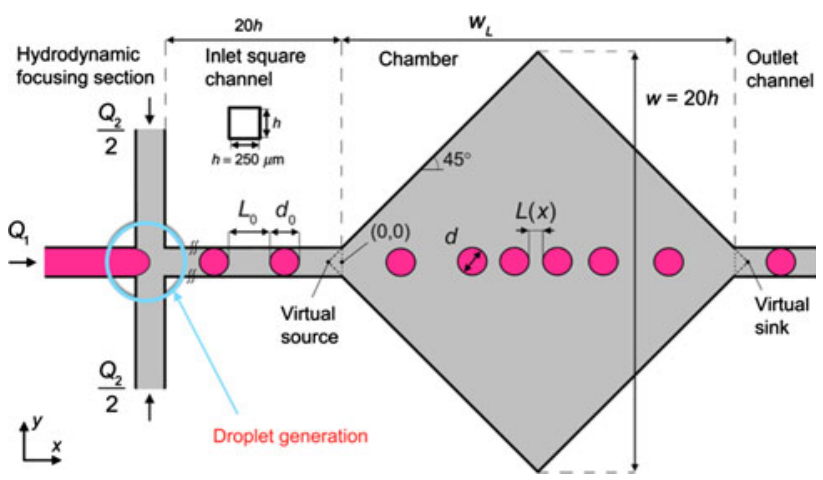

Fig. 1 Schematics of microfluidic module with focusing section, inlet square microchannel, chamber, and outlet channel (see text for details) 
Table 1 Properties of pairs of liquids

\begin{tabular}{|c|c|c|c|c|c|c|}
\hline Fluid pair & $L 1$ & $\eta_{1}(\mathrm{cP})$ & $L 2$ & $\eta_{2}(\mathrm{cP})$ & $\chi$ & $\gamma_{12}\left(\mathrm{mN} \mathrm{m}^{-1}\right)$ \\
\hline$G 1$ & WG 80 & 36 & PDMS & 0.82 & 44 & 33.8 \\
\hline$G 2$ & WG 92 & 272 & - & 19.0 & 14.3 & 30.4 \\
\hline$G 3$ & WG 80 & 36 & - & 4.59 & 7.8 & 33.7 \\
\hline G4 & - & 36 & - & 19.0 & 1.9 & 33.0 \\
\hline
\end{tabular}

Droplets are made of $L 1$ and the continuous phase is composed of $L 2$. The volume percentage of glycerol in the water/glycerol mixture (WG) is given for $L 1$. The viscosity ratio is $\chi=\eta_{1} / \eta_{2}$ and the interfacial tension is $\gamma_{12}$

production from droplet arrangement in the chamber. The reference point $(x, y)=(0,0)$ is set at the transition between the square and the diverging microchannels. In the chamber, single-phase flows at low Reynolds numbers can be described as a combination of a virtual point source and point sink separated by $w=20 h$. However, given the finite size of the inlet and outlet square channels of width $h$, the effective length of the microfluidic chamber is $w_{L}=19 h$.

Droplets are made of a mixture of water and glycerol (WG) and the continuous phase is composed of silicone oils (PDMS). Four fluid pairs with different viscosity ratios $\chi=\eta_{1} / \eta_{2}$ and similar interfacial tensions $\gamma_{12}$ are examined in the absence of surfactant, i.e., for a clean interface (Table 1). The viscosity of fluid $L 1$ is chosen large to examine the behavior of droplets made of high-viscosity fluids in bounded microflows. The interfacial tension $\gamma_{12}$ between liquids is measured using the combined capillary rise method (Rashidnia et al. 1992) and the viscosity of water-glycerol mixtures is measured using viscometer tubes. Since silicon and borosilicate glass have similar wetting properties, we measure the dynamic wetting properties of $L 1$ on borosilicate glass when immersed in a bath of L2 using a goniometer and a capillary tube connected to a syringe pump for droplet feeding. When the contact line velocity $V \rightarrow 0$, the advancing contact angle is $\theta_{\mathrm{A}} \approx 95 \pm 10^{\circ}$. Varying the speed of the contact line and fitting data with the hydrodynamic theory of dynamic contact angles (Blake 2006), $\theta=\theta_{\mathrm{A}}+a C a^{1 / 3}$ with $a$ being a constant, indicates that the advancing contact angle $\theta_{\mathrm{A}}$ tends to $180^{\circ}$ for capillary numbers $\mathrm{Ca}=\eta_{2} \mathrm{VI}$ $\gamma_{12} \sim 10^{-3}$. In this work, droplets are steadily generated above this critical capillary number and do not wet the walls of the inlet square microchannel. For all experiments, the Reynolds number $R e$ is calculated based on the viscosity of the external fluid and ranges between $8 \times 10^{-3}$ and 4 in the chamber.

\section{Droplet formation}

The initial morphology of the microfluidic emulsion is examined in the inlet square microchannel. Adjusting the flow rates $Q_{1}$ and $Q_{2}$ permits the control of droplet length $d_{0}$ and spacing $L_{0}$. Two important dimensionless quantities are the flow rate ratio $\varphi=Q_{1} / Q_{2}$ and the droplet concentration (or volume fraction) $\alpha_{1}=Q_{1} /\left(Q_{1}+Q_{2}\right)$. For dilute systems $\left(\alpha_{1} \leq 0.5\right)$ in the dripping regime, the droplet production mode is characterized by a pseudo-constant droplet size $d_{0} \sim h$ with large variation of the droplet spacing $L_{0}$. In contrast, the continuous microflow generation of concentrated emulsions $\left(\alpha_{1} \geq 0.5\right)$ is characterized by a droplet spacing that remains nearly constant $L_{0} \sim h$ and a wide change in droplet size $d_{0}$. Here, we focus on dilute systems as this regime allows for building elaborated droplet assemblies in the chamber. For large viscosity ratio $\chi \geq 22$, previous study on droplet formation in square hydrodynamic focusing sections (Cubaud and Mason 2008a) showed that the droplet size scales with $\alpha_{2} \mathrm{Ca}_{2}$ where $\alpha_{2}$ is the volume fraction of the continuous phase (i.e., $\left.\alpha_{2}=Q_{2} /\left(Q_{1}+Q_{2}\right)=1-\alpha_{1}\right)$ and $C a_{2}$ is the capillary number associated with the superficial velocity of the continuous phase at the junction (i.e., $C a_{2}=\eta_{2} Q_{2} /$ $\left.\left(\gamma_{12} h^{2}\right)\right)$. In the dilute regime, we measure the normalized droplet size $d_{0} / h$ as a function of $\alpha_{2} C a_{2}$ and find that the relationship $d / h=0.5\left(\mathrm{Ca}_{2} \alpha_{2}\right)^{-0.17}$ shows agreement with experimental data for $\varphi<0.8$ (Fig. 2a). We note a departure from this scaling for droplets having a low viscosity contrast with the external phase. Overall, experiments are conducted with a droplet size $d_{0} / h$ ranging between 0.7 and 1.8. Although the change in droplet size is relatively modest, it has implications on flow dynamics.

The droplet velocity in the square channel $V_{0}$ is measured from high-speed movies and compared with the multiphase flow superficial velocity $J_{0}=\left(Q_{1}+Q_{2}\right) / h^{2}$ as a function of the capillary number $C a=\eta_{2} J_{0} / \gamma_{12}$ in the square channel (Fig. 2b). Similar to the theory of elongated bubbles and droplets in circular and polygonal capillaries (Bretherton 1961; Ratulowski and Chang 1989; Wong et al. 1995), the droplet speed exceeds the superficial velocity and data are fit with the function $V_{0} / J_{0}=1+a C a^{2 / 3}$, with $a=2.5$. The scatter is due in part to the variety of droplet sizes obtained at similar capillary numbers. The droplet size and shape modify the drag force exerted by the external fluid but the general trend for small droplets compares well with that of elongated droplets. The multiphase flow production is steady when droplets are 

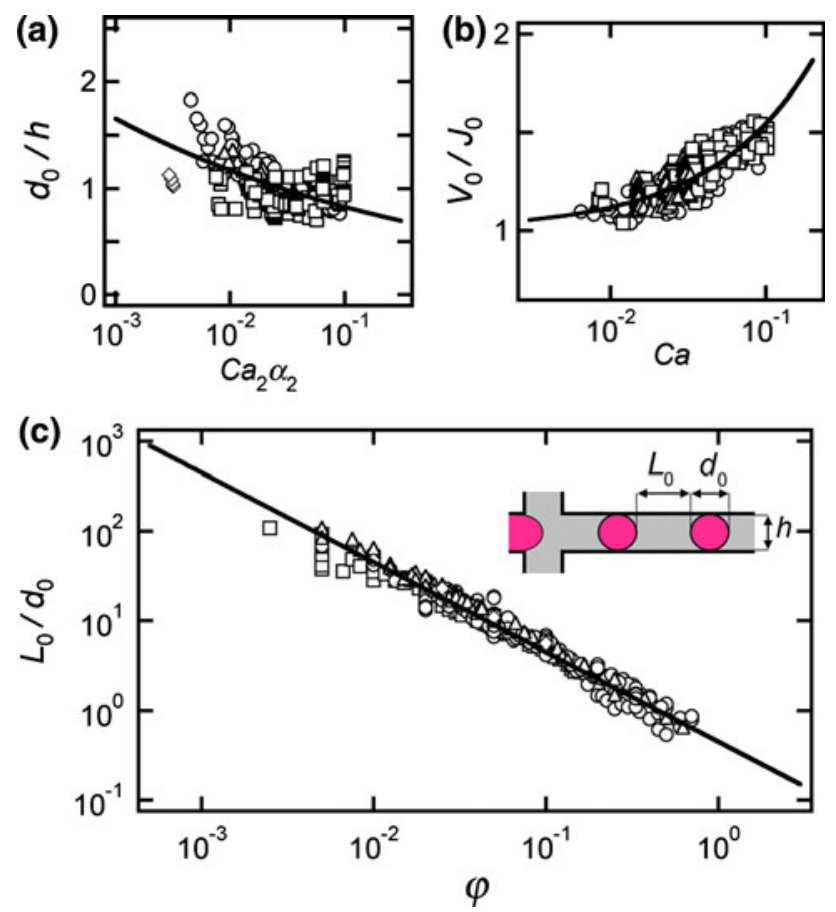

Fig. 2 Characteristics of microfluidic emulsions in the inlet square microchannel. Symbols of different fluid pairs: $G 1$ (diamond), $G 2$ (square), G3 (triangle), and G4 (circle). a Normalized droplet length $d_{0} / h$ as a function of $\mathrm{Ca}_{2} \alpha_{2}$, solid line: $d / h=0.5\left(\mathrm{Ca}_{2} \alpha_{2}\right)^{-0.17}$. b Droplet velocity $V_{0}$ normalized with total superficial velocity $J_{0}$ as a function of capillary number $\mathrm{Ca}$ in the square channel. Solid line: $V_{0} / J_{0}=1+2.54 \mathrm{Ca}^{2 / 3}$. c Initial distance between two consecutive droplets in the square channel normalized with droplet diameter $L_{0} / d_{0}$ as a function of flow rate ratio $\varphi$. Solid line: $L_{0} / d_{0}=0.45 \varphi^{-1}$

dynamically non-wetting the walls (i.e., for $\mathrm{Ca}>10^{-3}$ ). For larger capillary numbers $\mathrm{Ca} \sim 10^{-1}$, satellite droplets are observed during breakup. The sizes of satellite droplets,

(a)

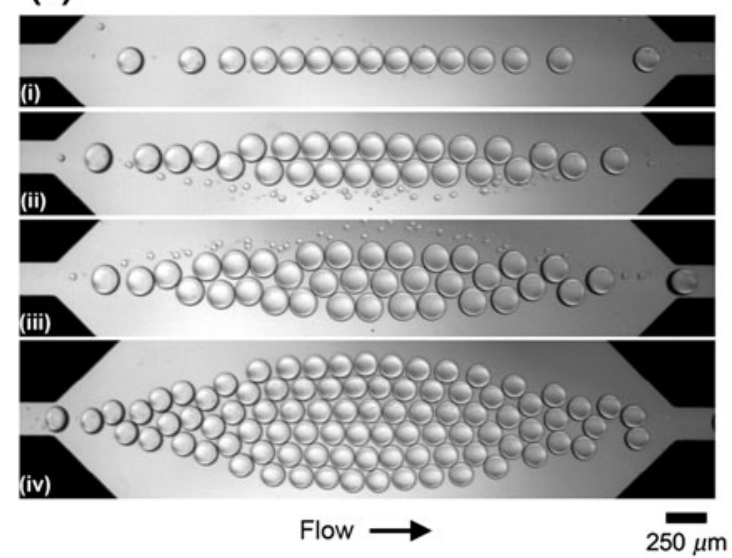

Fig. 3 a Formation of multiple layers in the microfluidic chamber, fluid pair $G 4$. Flow rates in $\mu \mathrm{l} / \mathrm{min}, Q_{2}=400(i)$ one layer, $Q_{1}=14$; (ii) two layers, $Q_{1}=30$; (iii) three layers, $Q_{1}=40$ and (iv) six layers formation $Q_{1}=85$. b General phase diagram with all fluid pairs, $G 1$ however, are negligible compared to the dimensions of the main droplets and such droplets do not significantly influence the system.

In the dilute emulsion regime, the distance between droplets $L_{0}$ can be controlled over several orders of magnitude. This property is advantageous for examining hydrodynamic coupling between droplets. In Fig. 2c, the droplet spacing normalized by the droplet size $L_{0} / d_{0}$ is measured as a function of the flow rate ratio $\varphi$. While the droplet size $d_{0}$ depends on both the volume fraction $\alpha_{2}$ (or flow rate ratio since $1 / \alpha_{2}=1+\varphi$ ) and the capillary number $\mathrm{Ca}_{2}$, mass conservation in segmented flow suggests a simple scaling: $L_{0} / d_{0} \sim Q_{2} / Q_{1}$, which is independent of the absolute flow velocity (i.e., the capillary number). Here, experimental data fit very well with the expression $L_{0} / d_{0}=0.45 \varphi^{-1}$. This property is shared between liquid/ liquid and liquid/gas microfluidic multiphase flows in the absence of significant compressibility effects (Sun and Cubaud 2011). The initial distance between two consecutive droplets $L_{0}$ is used as a control parameter to describe droplet arrangements in the microfluidic chamber.

\section{Microfluidic droplet sprays}

\subsection{Phase-diagram}

In the diverging-converging channel, droplets can form compact multi-layer flow arrangements (Fig. 3a). Droplets previously elongated in the square channel $\left(d_{0}>h\right)$ adopt a symmetric circular shape as they experience a change in confinement when they enter the chamber. On average, the number of droplets in the chamber remains constant since (b)

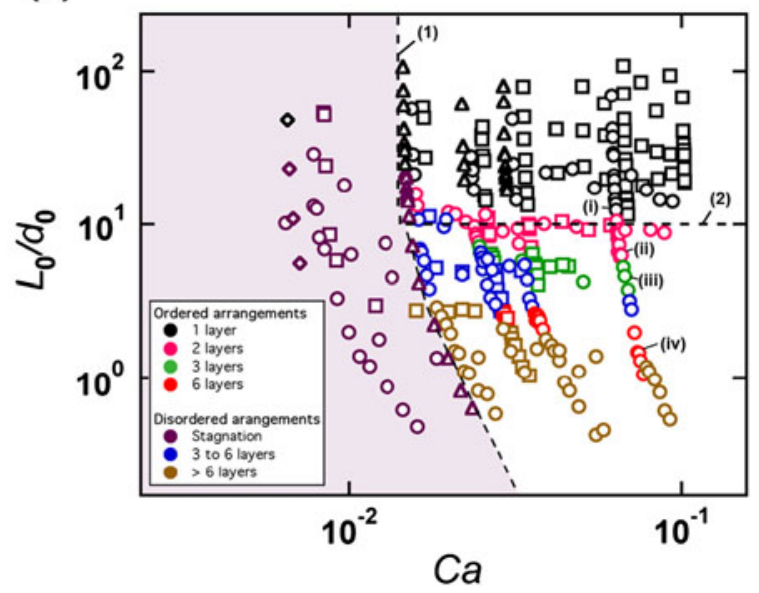

(diamond), G2 (square), G3 (triangle), and G4 (circle). Shaded region: stagnation of droplets at low capillary numbers; dashed-line (1) $C a=1.5 \times 10^{-2}$; dashed-line (2) $L_{0} / d_{0}=10$ 
the time-average number of incoming droplets is identical to the number of droplets exiting the chamber. The formation of regular and irregular packing structures depends on the initial droplet concentration in the square channel. For large initial spacing $\left(L_{0} / d_{0}>10\right)$, droplets form a single layer in the chamber. As $L_{0} / d_{0}$ is progressively lowered, ordered arrangements of two, three, or six layers of droplets are observed (Fig. 3a). To our surprise, no occurrence of four or five ordered layered arrangements is obtained. Rather, a complex transition regime is seen from three- to six-layered arrangements. Our cell shape and aspect ratio $(w / h=20)$ appear to prevent the formation of regular structures with more than six layers. While droplets are generally monodisperse, small size differences contribute to the formation of irregular layers.

A general phase-diagram of flow patterns obtained for all fluid pairs is displayed in Fig. 3(b). For low inlet capillary numbers $C a<1.5 \times 10^{-2}$, a few droplets attach to the top and bottom walls in the diverging channel. This stagnation regime is related to the dynamic wetting properties of our system. Indeed, in the center on the chamber, the superficial flow velocity is reduced by a factor $w / h=20$ compared to the square channel velocity $J_{0}$. Therefore, droplets can partially wet the glass substrate in the chamber for $C a / 20<10^{-3}$. In this case, the contact angle hysteresis yields a droplet adhesion force on the solid that is larger than the drag force exerted by the external fluid $L 2$. The critical value of the local capillary number $\left(\mathrm{Ca} \sim 10^{-3}\right)$ for droplet detachment from our surface compares well with previous work on bubbles adhesion in slit microchannels (Blackmore et al. 2001). The viscosity of the external phase $\eta_{2}$ also plays an important role in the formation of droplet assemblies. Over the range of parameters investigated, ordered layered arrangements are observed for fluid pairs $G 2$ and $G 4$, which have a relatively viscous continuous phase $\left(\eta_{2}=19 \mathrm{cP}\right)$. In contrast, for a less viscous external phase $\eta_{2}$, experiments with fluid pairs $G 1$ and $G 3$ yielded only one-layered arrangements since droplets in close proximity are found to rapidly coalesce, which significantly alters flow morphologies.

\subsection{Distance between droplets}

The distance between two consecutive droplets $L$ is an important factor governing flow assembly. In the chamber, $L$ is a function of the spatial coordinate $x$. One-row arrangements $(n=1)$ are characterized by a droplet trajectory following a straight line. In this regime, the continuous measurement of $L(\mathrm{x})$ is performed using spatiotemporal diagrams. Such diagrams are created from high-speed movies by plotting a line along the microchannel axis $x$ at $y=0$ and using the "reslice" function in ImageJ. Fortran routines are then implemented to extract the time and position of droplets. For a given droplet pair at the instant $t$, the rear position of the first droplet $x_{\mathrm{R}}(t)$ and the front position of the second droplet $x_{\mathrm{F}}(t)$ are used to compute $L(t)=x_{\mathrm{R}}(t)-x_{\mathrm{F}}(t)$. The spatial coordinate associated with $L$ corresponds to the average position of the droplet pair $x=\left[x_{\mathrm{R}}(t)+x_{\mathrm{F}}(t)\right] / 2$ (Fig 4a). Figure 4b displays the evolution of the droplet distance normalized by the initial distance $L / L_{0}$ in the square microchannel. This method shows that $L$ is minimal at the center of the cell, $L_{\mathrm{M}}=L\left(x=w_{\mathrm{L}} / 2\right)$, which corresponds to the transition between the diverging and the converging channel. In the very dilute regime, the normalized distance is seen to decrease in the inlet channel since the first droplet decelerates in the chamber, while the second droplet has a constant velocity the inlet channel. Using the constantspeed property in the inlet channel allows for calculating very large initial distance $L_{0}$ by extrapolating spatiotemporal diagrams for the dilute case. For a variety of fluid pairs and flow rates, we measure the minimal distance between droplets $L_{\mathrm{M}} / d_{0}$ as a function of the initial droplet train morphology factor $L_{0} / d_{0}$ and find that the curve $L_{\mathrm{M}} / d_{0}=5 \times 10^{-3}\left(L_{0} / d_{0}\right)^{1.6}$ fits data well (Fig. $4 \mathrm{c}$ ). The minimum distance is negligible for $L_{0} / d_{0}<10$. In this case, droplets are strongly coupled with one another and form multi-layer arrangements. Above this critical value, the minimum distance $L_{\mathrm{M}}$ monotonically increases with $L_{0}$ and droplets behave independent of one another. Our
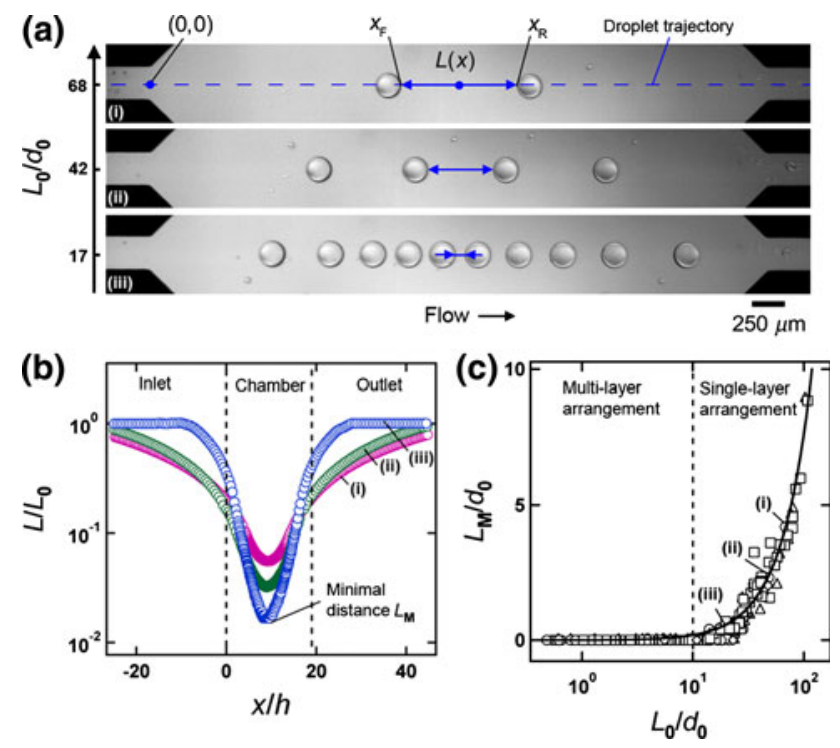

Fig. 4 a Evolution of the distance between two droplets $L(x)$ in the chamber for one-layer arrangement (very dilute emulsion), fluid pair $G 4$, flow rates in $\mu \mathrm{L} / \mathrm{min}$, fixed side flow rate $Q_{2}=400$ and varying $Q_{1}=2$, 4, and 10 (from top to bottom). b Droplet spacing $L$ normalized by initial spacing $L_{0}$ as a function of dimensionless position $x / h$. c Evolution of normalized minimal distance $L_{\mathrm{M}} / d_{0}$ between two droplets as a function of droplet train aspect ratio $L_{0} / d_{0}$, fluid pairs $G 1$ (diamond), G2 (square), G3 (triangle), and G4 (circle), solid line: $L_{\mathrm{M}} / d_{0}=5 \times 10^{-3}\left(L_{0} / d_{0}\right)^{1.6}$ 
experimental data suggest that the critical value for the crossover between collective and individual droplet behavior, $L_{0} / d_{0} \sim 10$, is imposed by the aspect ratio of the chamber $w / h=20$ since $d_{0} \sim h$ and $x_{\mathrm{M}}=w_{\mathrm{L}} / 2$.

\subsection{Envelope of droplet streams}

The microfluidic chamber offers the possibility to study the formation of compact arrays of droplets confined by the flow of the external fluid $L 2$. The dense stream of droplets or "spray" remains enclosed within an envelope of amplitude $A$ that typically increases with the dispersed phase concentration $\alpha_{1}$ (Fig. 5a). The evolution of the envelope amplitude $A(x)$ for each flow configuration is calculated from a composite image made by superimposing consecutive micrographs from a high-speed movie. The droplet lattice is widest in the center of the cell $A_{\max }=$ $A\left(x=w_{\mathrm{L}} / 2\right)$, similar to a stream made of continuous fluid (Darvishi and Cubaud 2011). This property results from mass conservation since the minimal droplet distance $L_{\mathrm{M}}$ is also found at this location. The shape of the envelope is determined by the chamber geometry. A typical experiment consists in fixing the side flow rate $Q_{2}$ and increasing

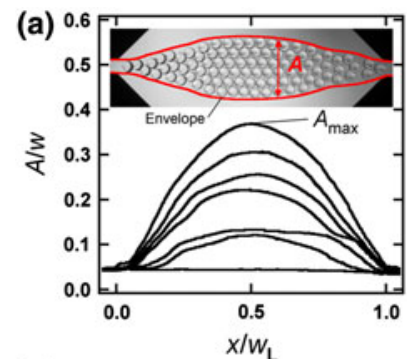

(c)
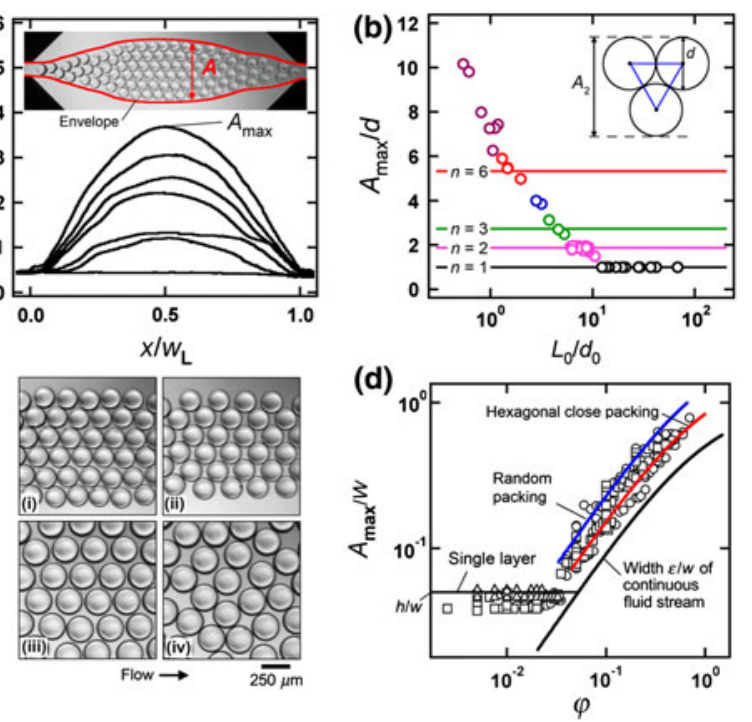

Fig. 5 a Spatial evolution of the envelope amplitude $A / w$ for fluid pair $G 4$ and fixed $Q_{2}=400 \mu \mathrm{L} / \mathrm{min}$, with $Q_{1}=120,100,85,4030$, $13 \mu \mathrm{L} / \mathrm{min}$ (from top to bottom). Inset: micrograph with superimposition of envelope amplitude. b Maximum amplitude normalized by droplet diameter in the chamber $A_{\max } / d$ as a function normalized distance between droplets $L_{0} / d_{0}$ with amplitude $A_{\mathrm{n}}$ associated with $n$ rows of hexagonal close packing of spheres between two plates (fluid pair $G 4$ with constant $Q_{2}=400 \mu \mathrm{L} / \mathrm{min}$ and varying $Q_{1}$ ). c Examples of droplet arrangements: (i) hexagonal close packing (ii) centered rectangular packing (iii) hexagonal dilute packing, (iv) random close packing. d Evolution of dimensionless maximum amplitude $A_{\max } / w$ as a function of flow rate ratio $\varphi$ with equivalent width $\varepsilon$ of a continuous fluid stream in the chamber, fluid pairs $G 1$ (diamond), G2 (square), G3 (triangle), and G4 (circle)
$Q_{1}$. In the one-layer arrangement, the maximal envelope amplitude is constant $A_{\max } \sim d$, where $d$ is the diameter of the droplet in the chamber (Fig. 5b). As $Q_{1}$ augments, $A_{\max }$ reaches various steps corresponding to different layered patterns. A collection of two-dimensional droplet lattices is observed, including hexagonal close packing, centered regular packing, hexagonal dilute packing, and random close packing (Fig. 5c). The most compact arrangement is obtained for hexagonal close packing and the amplitude $A_{\mathrm{n}}$ associated with $n$ rows of droplets is $A_{\mathrm{n}}=d[1+(n-1)$ $\left.(3 / 4)^{1 / 2}\right]$. The calculated amplitudes $A_{\mathrm{n}}$ are displayed in Fig. $5 \mathrm{~b}$ and overall show good agreement with the measured maximal amplitudes $A_{\max }$ associated with each row.

A comparative study is conducted between streams made of discrete droplets and streams made of continuous fluid. In a plane channel of width $w$, the thickness $\varepsilon$ of a hydrodynamically focused stream of miscible fluid having the same viscosity as the sheath fluid follows the expression $\varepsilon / w=\left(1+\varphi^{-1}\right)^{-1}$ (Cubaud and Mason 2008b). In Fig. 5d, we plot this relation for the continuous fluid thickness $\varepsilon / w$ along with the maximum amplitude $A_{\max } /$ $w$ of droplet streams as a function of the flow rate ratio $\varphi$. A major difference between the two cases occurs for low $\varphi$ since the amplitude $A_{\max }$ of the droplet stream remains constant for one-layer arrangements and is equal to $d \sim h=w / 20$ given the chamber aspect ratio. For larger droplet concentrations, however, a more direct comparison can be drawn between $A_{\max }$ and $\varepsilon$ using the packing factor $a$, which is defined as the fraction of volume occupied by droplets in a unit lattice structure. A two-dimensional hexagonal close arrangement of spheres of diameter $d \sim h$ has a packing factor $a=\left(\pi d^{3} / 12\right) /\left[(3)^{1 / 2} d^{3} /\right.$ $4] \approx 0.6$. Balancing the volume flow rate of a continuous stream $Q=V h \varepsilon$ with the effective flow rate of the stream made of droplet $Q_{\mathrm{d}}=V h A_{\max }$ such as $Q_{\mathrm{d}}=a Q$ yields $A_{\max }=a \varepsilon \approx 0.6 h\left(1+\varphi^{-1}\right)^{-1}$. This relation is plotted in Fig. $5 \mathrm{~d}$ and show good agreement with the smallest measured values of $A_{\max } / w$. The upper value of $A_{\max }$ appears to correspond to a lower packing factor $a \approx 0.4$ and is associated with random packing.

\subsection{Velocity and residence time in the chamber}

The multiphase superficial velocity $J$ is modified in the microfluidic chamber. The creeping flow situation (i.e., low Reynolds number $R e$ ) permits a simple expression for $J(\mathrm{x})$ using a source and a sink flow approximation. In the diverging channel $\left(x \leq w_{\mathrm{L}} / 2\right)$, the velocity decreases as $J=J_{0} /(2 x / h+1)$ until it reaches $J=J_{0} / 20$ for $x=w_{\mathrm{L}} / 2$ at the transition between the diverging and the converging channel. In the converging section $\left(x>w_{\mathrm{L}} / 2\right)$, the flow experiences a similar convective acceleration until $J=J_{0}$ for $x=w_{\mathrm{L}}$. To examine droplet motion in the chamber, the 
droplet velocity $V(x)$ is normalized by the initial droplet velocity $V_{0}$ in the square channel. For one-row arrangement, the droplet velocity is calculated from processing spatiotemporal diagrams (created from a straight line in $y=0)$ such as $V(x, t)=\mathrm{d} x / \mathrm{d} t$ where $x=\left(x_{\mathrm{F}}+x_{\mathrm{R}}\right) / 2$ with $x_{\mathrm{F}}$ being the front location and $x_{\mathrm{R}}$ the rear location of the same droplet. In multi-layer formation, the velocity and trajectory of each droplet are found using the "Manual Tracking" plug-in in ImageJ. Figure 6a shows a time series of the trajectories of six consecutive droplets for $n=6$. Droplets are periodically deposited into the upper and the lower branch similar to thread folding (Cubaud and Mason 2006; Chabert and Viovy 2008). Trajectories resemble that of the continuous phase streamlines and can be roughly modeled as circular arcs between a source and sink located inside the chamber at $x \approx h(i)$ and $x \approx w_{\mathrm{L}}-h$, respectively. In this example, the separation point for trajectories of the three droplets in the upper branch (ii) is located further downstream. A similar behavior is observed for the two droplets near the edge (iii). The normalized local
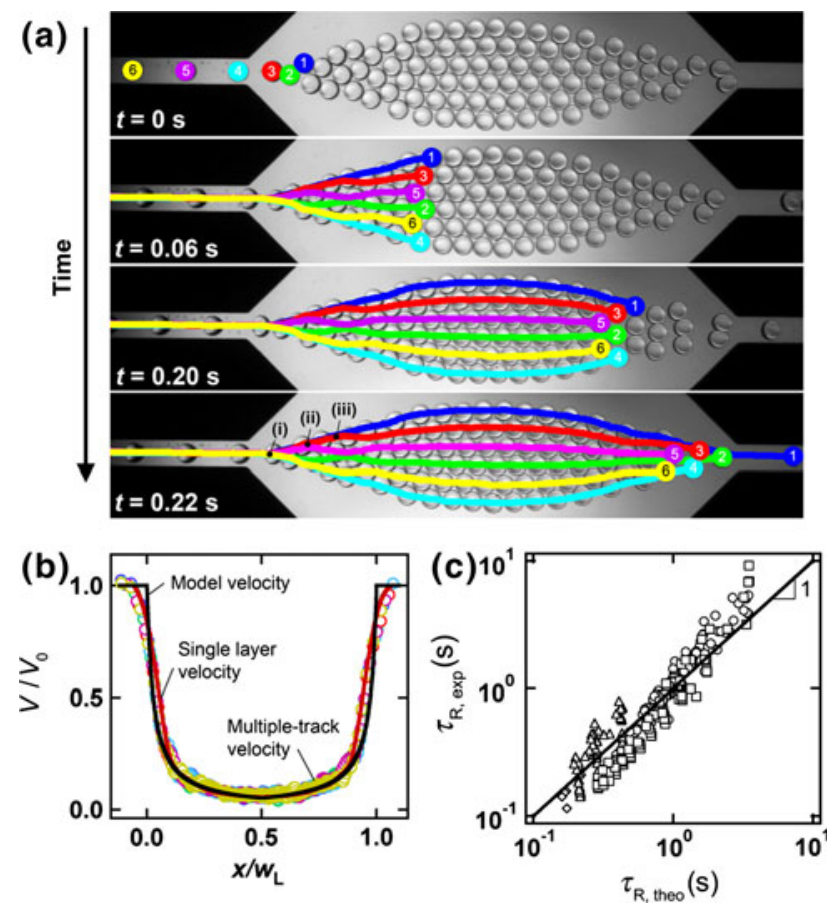

Fig. 6 a Temporal evolution of the trajectories of six successive droplets in a six-layer arrangement (fluid pair $G 4$, flow rates $Q_{1}=80$ and $Q_{2}=400 \mu \mathrm{L} / \mathrm{min}$ ) (i) separation point for trajectories between the upper and lower branches, (ii) separation point for three droplets in the upper branch, (iii) separation point for two droplets at the edge of the upper branch. b Evolution of droplet velocity $V$ normalized by inlet velocity $V_{0}$ as a function of location $x / w_{\mathrm{L}}$ with model velocity, single-layer (fluid pair: $G 4$, flow rates $Q_{1}=6$ and $Q_{2}=200 \mu \mathrm{L} / \mathrm{min}$ ) and multi-layer velocities corresponding to (a). c Measured residence time $\tau_{\mathrm{R} \text { exp }}$ versus theoretical residence time $\tau_{\mathrm{R} \text {,theo, solid line: }}$ $\tau_{\mathrm{R}, \exp }=\tau_{\mathrm{R} \text {,theo }}$, fluid pairs $G 1$ (diamond), G2 (square), G3 (triangle), and $G 4$ (circle) velocity of each droplet $V / V_{0}$ is computed from experimental movies and show good agreement with the model velocity and the velocity extracted for $n=1$ (Fig 6b). In the region where $J$ changes significantly, droplets tend to adopt a smoother velocity that our simple prediction. An intriguing effect of droplet assembly is that the velocity of droplets near the envelope edge is similar to the one associated with droplets in the center of the envelope with $V / V_{0} \approx J / J_{0}$. Indeed, the distance traveled by droplets near the edge only slightly surpasses the distance in the center of the chamber at $y=0$. As a result of this property, droplet lattices flow such as a solid crystal of droplets having a velocity equivalent to a one-row arrangement. Droplets are found to exit the channel in the same order of entrance.

Using the symmetry between the diverging and converging channels, the residence time $\tau_{\mathrm{R} \text {,theo }}$ is calculated by integrating $\mathrm{d} \tau_{\mathrm{R}}=[2 / J(x)] \mathrm{d} x$ for $x$ varying from 0 to $w_{\mathrm{L}} / 2$ and yields $\tau_{\mathrm{R} \text {,theo }}=w_{\mathrm{L}} w h /\left(Q_{1}+Q_{2}\right)$. For a variety of droplet arrangements, the residence time $\tau_{\mathrm{R} \text {,exp }}$ is experimentally measured from movies and overall shows good agreement with $\tau_{\mathrm{R} \text {,theo }}$ (Fig. 6c). Small residence times are typically associated with large capillary number $C a$ and $\tau_{\mathrm{R} \text {,exp }}$ are lower than the calculated values indicating that droplets travel faster than $J$ as shown in Fig. 2b. In general, this system allows for modulating the residence time in the chamber with flow rates of injection regardless of the flow morphology. The residence time is an important quantity for estimating coalescence events between droplets.

\subsection{Droplet coalescence}

Microfluidic chambers are useful for merging two consecutive droplets in mixing applications (Lai et al. 2009; Shemesh et al. 2011). When two droplets make direct contact, capillary forces join them together over a short period of time, which defines the coalescence time-scale $\tau_{\mathrm{c}}<10^{-3} \mathrm{~s}$. This mechanism is nearly spontaneous for two droplets in close proximity in air. In a viscous environment, however, two droplets in apparent contact do not necessarily rapidly coalesce even in the absence of surfactant. Indeed, one needs to consider the draining time required for displacing the thin liquid film between two droplets. Measurements of draining times are relatively scarce in the literature due to the difficulty in manipulating individual droplets in a viscous matrix (Yang et al. 2001; Yoon et al. 2005). Here, different sets of experiments are conducted for each fluid pair by fixing $Q_{2}$ and progressively increasing $Q_{1}$. For large initial distances $L_{0} / d_{0}>30$, no coalescence is observed since the minimal distance between droplet is always $L_{\mathrm{M}}>0$. As $L_{0} / d_{0}$ decreases (or $Q_{1}$ increases), however, various coalescence regimes are observed. The fusion of two droplets produces a larger droplet, which is displaced at a lower speed due to its 
increased drag (Blackmore et al. 2001). For low capillary numbers and $n=1$, incoming droplets merge with larger front droplets and steady regimes where fused droplets made of three or four initial droplets are observed (Fig. 7a). Over the range of parameters investigated, droplets made of more than five initial droplets become stagnant and produce the "stagnation regime" for very low $\mathrm{Ca}$. For moderate capillary numbers, steady binary droplet coalescence regimes characterized by leading droplets made of two initial droplets are also observed (Fig. 7b). The slow motion of a leading droplet in one-row arrangement can lead to the buckling of the droplet chain, as indicated by the arrows in Fig. 7b. For large capillary numbers, although coalescence events do occur, they remain relatively rare. Using our experimental protocol, we progressively decrease $L_{0} / d_{0}$ and note the critical value where the first droplet coalescence is observed as a function of the capillary number $C a=\eta_{2} J_{0} / \gamma_{12}$ (Fig. 7c). Experimental data show that the critical $L_{0} / d_{0}$ decreases with $C a$ according to $L_{0} / d_{0}=4.8 \mathrm{Ca}^{-0.32}$. This finding gives evidence that the critical droplet concentration $\alpha_{1 \mathrm{C}}$ for coalescence in a microfluidic chamber increases with the capillary number according to $\alpha_{1 \mathrm{C}} \approx\left(1+10.7 \mathrm{Ca}^{-0.32}\right)^{-1}$. In other words, the stability of dilute emulsions in microgeometries improves with the speed of injection. The draining time $\tau_{\mathrm{D}}$ required for two droplets to coalesce is measured by counting the number of frames in a movie between the first
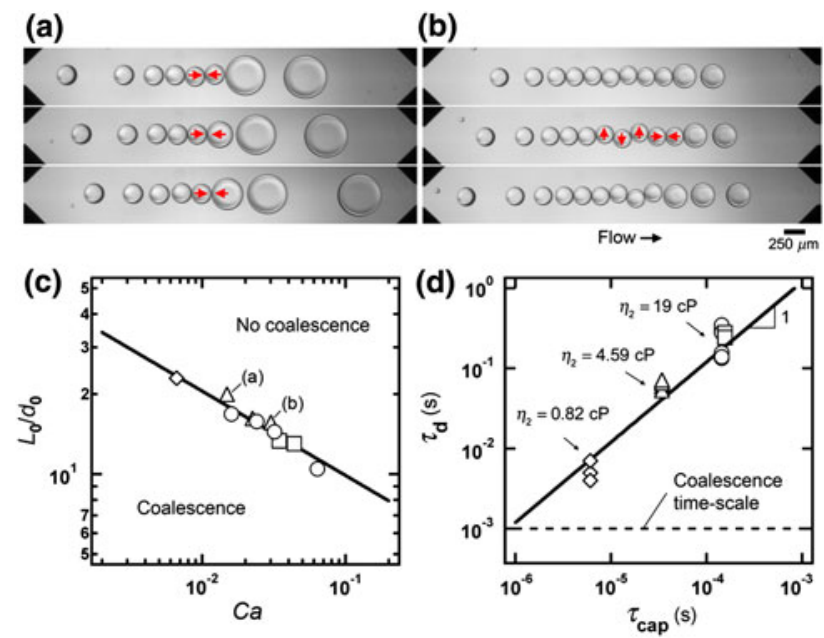

Fig. 7 Onset of coalescence in the microfluidic chamber. Time-series of successive droplet coalescences for one-row arrangement $(n=1)$, fluid pair $G 3$ : a low capillary number $C a \approx 1.5 \times 10^{-2}, \Delta t=$ $48 \mathrm{~ms}$; b moderate capillary number $C a \approx 3 \times 10^{-2}, \Delta t=9 \mathrm{~ms}$. Arrows show next droplet coalescence and buckling of droplet chain. c Diagram of first coalescence observed as $L_{0} / d_{0}$ is progressively decreased, fluid pairs $G 1$ (diamond), G2 (square), G3 (triangle), and $G 4$ (circle), solid line: $L_{0} / d_{0}=4.8 \mathrm{Ca}^{-0.32}$. d Comparison between measured draining time $\tau_{\mathrm{D}}$ and calculated viscous capillary time-scale $\tau_{\text {cap }}$, solid line $\tau_{\mathrm{D}}=10^{3} \tau_{\text {cap }}$ contact and coalescence (Fig. 7d). Data suggest that the draining time $\tau_{\mathrm{D}}$ is proportional to the viscous-capillary time-scale $\tau_{\text {cap }}=\eta_{2} h / \gamma_{12}$ with a functional relationship such as $\tau_{\mathrm{D}} \sim 10^{3} \tau_{\text {cap }}$. Experiments conducted at different injection speeds do not show any particular influence on the draining time $\tau_{\mathrm{D}}$ as would be expected for creeping flows. This work shows that the droplet viscosity $\eta_{1}$ does not play a significant role in triggering coalescence processes. In contrast, the influence of the absolute viscosity of the external phase $\eta_{2}$ is paramount. Overall, a method for predicting coalescence processes consists in comparing the draining time $\tau_{\mathrm{D}}$ with the droplet residence time $\tau_{\mathrm{R}}$ in the chamber: for $\tau_{\mathrm{D}}<\tau_{\mathrm{R}}$ coalescence is significant while for $\tau_{\mathrm{D}}>\tau_{\mathrm{R}}$ droplets behave as solid spheres.

Coalescence processes are known to introduce significant structural rearrangements in concentrated dispersions (Larson 1999). Here, in one-layer arrangement, the outcome of recombination consists in the formation of a row of larger droplets in the converging section. For multi-layer arrangements, a variety of situations are observed as a function of flow conditions. The actual merging of two adjacent droplets typically introduces local transient flows around the droplets that alter interstitial spaces between neighboring droplets, which may trigger their fusion similar to a chain reaction. Figure 8a displays the time-series of a coalescence cascade in two-row arrangements. Two droplets in close proximity fuse and create a larger, slow moving droplet that collects new incoming droplets. For larger $n$, the position of surrounding droplets is directly affected by a single coalescence event. For $n=3$, the merging of two droplets in the center row can produce a larger droplet with eight neighboring droplets circularly aligned (Fig. 8b). Coalescence avalanches are more likely to occur in compact arrangements with $n=6$. For
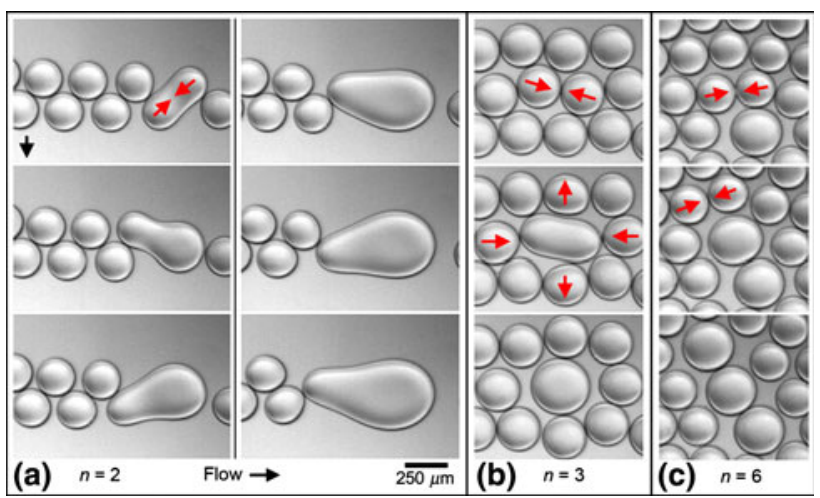

(a) $n=2$ Flow $\rightarrow$ $2 \overline{250 \mathrm{~m}}$ (b) $n=3$ (c) $n=6$

Fig. 8 Examples of droplet rearrangement processes due to coalescence, fluid pair $G 4$. a Coalescence cascade in a two-row arrangement ( $n=2$ ), flow rates $Q_{1}=25$ and $Q_{2}=400 \mu \mathrm{L} / \mathrm{min}$. b Droplet merging in a three-row arrangement $(n=3)$, flow rates $Q_{1}=50$ and $Q_{2}=400 \mu \mathrm{L} / \mathrm{min}$. c Coalescence avalanche in a six-row arrangement $(n=6), Q_{1}=41$ and $Q_{2}=200 \mu \mathrm{L} / \mathrm{min}$ 
instance, the merging of two droplets in Fig. 8c induces the coalescence of two other droplets in the immediate vicinity of the first coalescence. In turn, the second coalescence event displaces nearby droplets and generates a third coalescence. Similar coalescence fronts were recently observed for concentrated microfluidic emulsions (Bremond et al. 2011). Overall, our study shows that although the coalescence phenomenon may appear as a random process, it can be manipulated with the convective timescale (i.e., residence time of droplets) in microgeometries.

\section{Conclusion}

In this study, the flow behavior of a stream of droplets in a microfluidic chamber is quantitatively discussed. Four immiscible fluid pairs having different viscosities and similar interfacial tensions are examined. Generating droplets at the junction of a focusing section permits the manipulation of the incoming monodisperse droplet train aspect ratio $L_{0} / d_{0}$ with the injection flow rates. Fluid flow rates are selected to produce droplets having a size similar to that of the microchannel height $h$. First, we show that the initial droplet train aspect ratio in the square channel is inversely proportional to the flow rate ratio such as $L_{0} / d_{0}=0.45 \varphi^{-1}$. The resulting flow patterns in the chamber consist in stagnation flow for low capillary number $C a<1.5 \times 10^{-2}$ and one-row arrangement of droplets for $L_{0} / d_{0}>10$. As the initial distance between droplets decreases, compact droplet lattices having different numbers of rows $n$ are observed. For dilute emulsions, the minimal distance between droplets $L_{\mathrm{M}}$ is found in the middle of the chamber and can be fit with $L_{\mathrm{M}} / d_{0}=5 \times 10^{-3}\left(L_{0} / d_{0}\right)^{1.6}$. The maximal amplitude of the droplet school envelope $A_{\max }$ is compared with the spread of a continuous fluid focused by a sheath fluid taking into account the packing factor $a$ of solid spheres between two plates according to $A_{\max } \approx a h\left(1+\varphi^{-1}\right)^{-1}$. The width of the droplet stream agrees well with a packing factor $a$ ranging between two-dimensional hexagonal close packing $(a \approx 0.6)$ and an arbitrary random packing factor $(a \approx 0.4)$. The evolution of the droplet velocity in the channel compares with the local multiphase superficial velocity. In particular, we show that droplets are displaced at the same velocity in compact arrangement. In addition, measured droplet residence times in the cell are found to follow the theoretical relationship $\tau_{\mathrm{R} \text {,theo }}=w_{\mathrm{L}} w h /$ $\left(Q_{1}+Q_{2}\right)$. Finally, the relationship between droplet concentration and the injection capillary number for the onset of droplet coalescence in a pore is established. We demonstrate that the relevant factor for determining the onset of coalescence is the external phase viscosity $\eta_{2}$ and experimental correlations suggest that the maximal droplet concentration before coalescence is $\alpha_{1 \mathrm{C}} \approx\left(1+10.7 \mathrm{Ca}^{-0.32}\right)^{-1}$. Our experimental findings show that the film draining time $\tau_{\mathrm{D}}$ between droplets in apparent contact is proportional with the viscous-capillary time scale, $\tau_{\mathrm{D}} \sim 10^{3} \tau_{\text {cap }}$.

Overall, this study shows the possibility to control coalescence processes with flow rates of injection in microchannels. In the absence of coalescence and for moderate capillary numbers, droplets can be simply modeled as solid spheres. Numerical simulations of droplets flows in constrained geometries would provide additional insights for modeling immiscible multiphase flows in natural porous media, such as the ones encountered in oil reservoirs. Our study of droplet behavior in a simplified pore model highlights the interplay between droplet hydrodynamic coupling and coalescence phenomena. In microfluidic networks, a simple practical solution for preventing droplet coalescence in the absence of stabilizing agents (i.e., surfactants) is to use an external phase having a large viscosity coefficient in conjunction with large flow rates of injection.

Acknowledgments The authors have appreciated stimulating discussions with Eric Brouzes. This material is based upon work supported by the National Science Foundation under Grant No. CBET-0932925.

\section{References}

Aarts DGAL, Lekkerkerker HNW (2008) Droplet coalescence: drainage, film rupture and neck growth in ultralow interfacial tension systems. J Fluid Mech 606:275-294

Anna SL, Bontoux N, Stone HA (2003) Formation of dispersions using "flow focusing" in microchannels. App Phys Lett 82:364-366

Bear J (1972) Dynamics of fluids in porous media. Elsevier, New York

Blackmore B, Li D, Gao J (2001) Detachment of bubbles in slit microchannels by shearing flow. J Colloid Int Sci 241:514-520

Blake TD (2006) The physics of moving contact lines. J Colloid Int Sci 299:1-13

Boukellal H, Selimovic S, Jia Y, Cristobal G, Fraden S (2009) Simple, robust storage of drops and fluids in a microfluidic device. Lab Chip 9:331-338

Bremond N, Thiam AR, Bibette J (2008) Decompressing emulsion droplets favor coalescence. Phys Rev Lett 100:024501

Bremond N, Doméjean H, Bibette J (2011) Propagation of drop coalescence in a two-dimensional emulsion: a route toward phase inversion. Phys Rev Lett 106:214502

Bretherton FP (1961) The motion of bubbles in tubes. J Fluid Mech 10:166

Brouzes E, Medkova M, Savenelli N, Marran D, Twardowski M, Hutchison JB, Rothberg JM, Link DR, Perrimon N, Samuels ML (2009) Droplet microfluidic technology for single-cell highthroughput screening. Proc Natl Acad Sci USA 106:1419514200

Chabert M, Viovy JL (2008) Microfluidic high-throughput encapsulation and hydrodynamic self-sorting of single cells. Proc Natl Acad Sci USA 105:3191-3196

Cottin C, Bodiguel H, Colin A (2011) Influence of wetting conditions on drainage in porous media: a microfluidic study. Phys Rev E 84:026311 
Cubaud T (2009) Deformation and breakup of high-viscosity droplets with symmetric microfluidic cross flows. Phys Rev E 80:026307

Cubaud T, Mason TG (2006) Folding of viscous threads in diverging microchannels. Phys Rev Lett 96:114501

Cubaud T, Mason TG (2007) A microfluidic aquarium. Phys Fluids 19:091108

Cubaud T, Mason TG (2008a) Capillary threads and viscous droplets in square microchannels. Phys Fluids 20:053302

Cubaud T, Mason TG (2008b) Formation of miscible fluid microstructures by hydrodynamic focusing in plane geometries. Phys Rev E 78:056308

Darvishi S, Cubaud T (2011) Lubrication of highly viscous core-annular flows in microfluidic chambers. J Fluids Eng 133:031203

Dreyfus R, Tabeling P, Willaime H (2003) Ordered and disordered patterns in two-phase flows in microchannels. Phys Rev Lett 90:144505

Eggers J (1997) Nonlinear dynamics and breakup of free-surface flows. Rev Mod Phys 69:865-929

Gu H, Duits MHG, Mugele F (2011) Droplets formation and merging in two-phase flow microfluidics. Int J Mol Sci 12:25722597

Hashimoto M, Garstecki P, Stone HA, Whitesides GM (2008) Interfacial instabilities in a microfluidic Hele-Shaw cell. Soft Matter 4:1403-1413

Hatch AC, Fisher JS, Pentoney SL, Yang DL, Lee AP (2011) Tunable 3D droplet self-assembly for ultra-high-density digital microreactor arrays. Lab Chip 11:2509-2517

Jones AF, Wilson SDR (1978) The film drainage problem in droplet coalescence. J Fluid Mech 87:263-288

Lai A, Bremond N, Stone HA (2009) Separation-driven coalescence of droplets: an analytical criterion for the approach contact. J Fluid Mech 632:97-107

Larson RG (1999) The structure and rheology of complex fluids. Oxford University Press, New York

Leal-Calderon F, Schmitt V, Bibette J (2007) Emulsion science: basic principles. Springer Science + Business Media LLC, New York

Link DR, Anna SL, Weitz DA, Stone HA (2004) Geometrically mediated breakup of drops in microfluidic devices. Phys Rev Lett 92:054503
Rabe C, Malet J, Feuillebois F (2010) Experimental investigation of water droplet binary collisions and description of outcomes with a symmetric Weber number. Phys Fluids 22:047101

Rashidnia N, Balasubramaniam R, Del Signore D (1992) Interfacial tension measurement of immiscible liquids using a capillary tube. AIChE J 38:615-618

Ratulowski J, Chang H-C (1989) Transport of gas bubbles in capillaries. Phys Fluids A 1:1642

Schneider TM, Mandre S, Brenner MP (2011) Algorithm for a microfluidic assembly line. Phys Rev Lett 106:094503

Schultz KM, Furst EM (2011) High-throughput rheology in a microfluidic device. Lab Chip 11:3802

Shemesh J, Nir A, Bransky A, Levenberg S (2011) Coalescenceassisted generation of single nanoliter droplets with predefined composition. Lab Chip 11:3225-3230

Sun R, Cubaud T (2011) Dissolution of carbon dioxide bubbles and microfluidic multiphase flows. Lab Chip 11:2924

Tan YC, Ho YL, Lee AP (2007) Droplet coalescence by geometrically mediated flow in microfluidic channels. Microfluid Nanofluid 3:495

Teh SY, Lin R, Hung LH, Lee AP (2008) Droplet microfluidics. Lab Chip 8:198-220

Thorsen T, Roberts RW, Arnold FH, Quake SR (2001) Dynamic pattern formation in a vesicle-generating microfluidic device. Phys Rev Lett 86:4163

Wong H, Radke CJ, Morris S (1995) The motion of long bubbles in polygonal capillaries. Part 1. Thins films. J Fluid Mech 292:71

Wu M, Cubaud T, Ho C-M (2004) Scaling law in liquid drop coalescence driven by surface tension. Phys Fluids 16:L51

Yang H, Park CC, Hu YT, Leal LG (2001) The coalescence of two equal-sized drops in a two-dimensional linear flow. Phys Fluids 13:1087-1106

Yoon Y, Borrell M, Park CC, Leal LG (2005) Viscosity ratio effects on the coalescence of two equal-sized drops in a twodimensional linear flow. J Fluid Mech 252:355-379 